\title{
Urgences
}

\section{Il était une fois, ou deux, ou trois - ou les aventures d'un timbre-poste qui refusait d'être perdu}

\section{Renald Bérubé}

Numéro 32, mai 1991

Lectures de bandes dessinées

URI : https://id.erudit.org/iderudit/025653ar

DOI : https://doi.org/10.7202/025653ar

Aller au sommaire du numéro

Éditeur(s)

Urgences

ISSN

0226-9554 (imprimé)

1927-3924 (numérique)

Découvrir la revue

Citer cet article

Bérubé, R. (1991). Il était une fois, ou deux, ou trois - ou les aventures d'un timbre-poste qui refusait d'être perdu. Urgences, (32), 118-122.

https://doi.org/10.7202/025653ar d'utilisation que vous pouvez consulter en ligne.

https://apropos.erudit.org/fr/usagers/politique-dutilisation/ 
C'est le 22 mars 1991, à la Grange-théâtre du Bic, dans le cadre d'un événement multidisciplinaire intitulé Les eaux de mars: un jour à rompre les silences que le « document * qui suit a été lu.

Une vingtaine de textes - poèmes, fictions, constats, témoignages -, des voix, de la musique, de la chorégraphie.

L'idée d'un tel spectacle est venue à Louise Beauchamp, Jean-Marc Bélanger, Anne-Marie Clément et Luc Lavallée en décembre 1990, sur le coup, en quelque sorte, de la fermeture de la station télé de Radio-Canada à Rimouski.

À rappeler la circonstance, on fait surgir l'enjeu.

A. G.

\section{II était une fois, ou deux, ou trois - ou les aventures d'un timbre-poste qui refusait d'être perdu}

Allons, courage, vieil enfant [...], repousse le miel et le mièvre, l'innocence a besoin de ta hargne, comme Dieu du diable [...]. Descends encore une fois dans la mine désaffectée, peut-être y dort quelque filon, quelque pépite oubliée. Ne résiste plus, ouvre-toi, laisse déferler le flot qui monte du pays natal [...].

Gérald Tougas, La mauvaise foi ${ }^{1}$

1 Gérald Tougas, La mauvaise foi, coll. « Littérature d'Amérique ", Montréal, Québec/Amérique, 1990, p. 33. 
Il était une fois dans quelques petites villes - car sa famille déménageait et lui-même voyageait - de l'état très reculé (très deep) du Mississippi, un jeune homme qui rêvait de devenir écrivain et qui travaillait à la réalisation de ce rêve. Quelque trente années et un peu plus avant la naissance du jeune homme, son coin de pays natal mississippien avait connu les dévastations d'une guerre fratricide dont la chronique alimentait encore et toujours les récits locaux; au moment où il atteignait lui-même ses trente années d'âge, en 1927 disons, le jeune homme, qui avait alors beaucoup voyagé en USA et en Europe, avait déjà fait paraître divers textes, plus un recueil de poèmes et deux romans. Mais ce n'est qu'à l'occasion de son troisième roman, terminé cette même année de ses trente ans, qu'il devait découvrir quel serait le sujet, le lieu d'ancrage véritable de l'ensemble de son œuvre. Trente ans ou presque plus tard, en 1956 disons, le jeune homme un peu vieilli devait s'en expliquer ainsi à l'occasion d'une interview, genre qui lui semblait un mal nécessaire auquel sa célébrité le condamnait dorénavant à se plier sans trop de mauvaise grâce malgré tout. Le vieil enfant disait donc:

A partir de Sartoris, j'ai découvert que mon petit coin de terre natale, grand comme un timbre-poste, était un sujet valable, que je ne vivrais jamais assez longtemps pour l'épuiser et qu'en sublimant la réalité, j'aurais l'entière liberté d'employer au maximum le talent que je pouvais avoir. Cela m'ouvrit une mine d'or de personnages, aussi créai-je un univers bien à moi. Je peux faire vivre ces gens, tout comme si j'étais Dieu, non seulement dans l'espace, mais aussi dans le temps. [...] J'aime à me représenter le monde que j'ai créé comme une sorte de clé de voûte de l'univers; aussi minuscule soit-elle, si on la retirait, c'est l'univers entier qui s'écroulerait. ${ }^{2}$

En trente romans et autres textes environ, notre auteur n'allait cesser d'explorer le temps et l'espace de son "petit coin de terre natale" -2400 milles carrés en l'état du Mississippi, répartis autour de la petite ville universitaire d'Oxford ou de Jefferson, selon qu'on adopte le cadastre civil ou romanesque. Timbre-poste en effet, qui allait pourtant devenir une clé de voûte de la renaissance de tout le Sud des

2 Dans Romanciers au travail (traduction de Jean René Major, préface de Jacques Borel), coll. * Témoins ", Paris, Gallimard, 1967, p. 20. 
États-Unis et qui allait mettre ces lieux, et aléatoirement notre auteur qui s'en défendait farouchement, sur la carte du monde. Car l'auteur de Sartoris avait été lauréat du prix Nobel de littérature en 1950; comme quoi habiter ou décrire une région appelée par les autres périphérique, marginale ou éloignée n'est en rien un empêchement. Personne n'habite le monde; chacun et chacune habite un lieu précis qui peut aussi être le centre d'un et du monde.

Il était une fois, donc, l'œuvre de William Faulkner, puisque tel est, vous le savez bien, le nom de l'auteur de Sartoris. Et l'œuvre de Faulkner, dite aujourd'hui universelle, a trouvé son inspiration en un petit coin de terre natale de 2400 milles carrés, grand comme un timbre-poste. Dans les circonstances que nous connaissons, il est difficile de résister à la tentation d'ajouter ceci: l'arrière-grand-père de Faulkner avait autrefois construit un chemin de fer ensuite perdu par la famille, et Faulkner lui-même avait jadis brièvement travaillé à l'Université du Mississippi comme préposé à la poste. Via Rail et Postes Canada sévissant parfois dans nos parages, nous pouvons donc, même dans les détails, lire l'aventure faulknérienne comme emblématique et exemplaire. Car...

Car il était une fois une région grande comme plusieurs timbres-poste - et quelle que fút par ailleurs la tête couronnée apparaissant le plus souvent sur lesdits timbresposte - que les Postes de son pays éprouvaient de moins en moins le souci de rejoindre. Lisolant, lui rendant les communications difficiles, ces Postes et autres sociétés couronnées la refoulaient sur elle-même, l'éloignaient, la rapetissaient. Presque. Cette région, dorénavant, risquait de devenir le réceptacle des images des autres sans pouvoir efficacement transmettre la sienne. Et pourtant.

Et pourtant cette région, pour le littéraire acceptant volontiers de descendre dans la mine qu'on croyait désaffecter, cette région a fourni et continue de fournir à la littérature francophone de son pays des œuvres fort importantes: celles de l'abbé Casgrain, de Joseph-Charles Taché, de Jovette Bernier, de Victor-Lévy Beaulieu, de Madeleine Gagnon, de Noël Audet, de Paul Chanel Malenfant, de Marie Bélisle, de Louise Beauchamp. Pour ne donner que quelques noms; pour donner le nom de voix provenant du seul domaine littéraire. Car Radio-Canada, autre société couronnée, sait bien les voix 
que cette région a fournies ou a formées pour son réseau dorénavant tronqué. Dans cette région où la nature a malgré tout conservé des droits, la culture et l'enseignement ont aussi fleuri, Olivar Asselin et Gérard Filion pouvant en témoigner. Comment expliquer alors que la ville où ceux-ci étudièrent et où se trouvent depuis vingt ans et plus un cégep et une université, que cette ville ne fasse qu'achever la construction d'une bibliothèque décente, ne dispose toujours pas d'une salle de spectacles et ne dispose que d'un musée bien exigu? La mémoire et l'avenir ont des droits semblables qui relèvent du même acharnement à vivre et à se développer selon soi-même et le lieu de son timbre-poste; la région qui a su engendrer des entreprises dont Québec-Téléphone et l'Hydro-Québec, aujourd'hui, assurent la continuité; la région où, malgré tout, nature et culture ont réussi à cohabiter cette région, la devise du Québec étant "Je me souviens", doit se rappeler son passé forestier, dirait Joseph-Charles Taché, et ne pas laisser se dilapider son capital culturel comme fut dilapidé son héritage de forêts. Car...

Car il était une fois, la dernière pour ce soir, celui qui s'adresse à vous. Il est né en ce qu'il nomme la Salmonie, c'est-à-dire dans le village de Lac-au-Saumon dans la vallée de la Matapédia. Il a vu, alors qu'il étudiait à Montréal et ailleurs, le village de son enfance se transformer: des moulins à bois ont cessé leurs activités, des terres ont cessé d'être cultivées. La beurrerie où il a apporté tant de bidons de crème depuis la ferme de ses parents et celles des autres cultivateurs des alentours, où il a appris comment le lait, après être devenu crème, devenait beurre, cette beurrerie s'est transformée en maison à logements. Il a vu aussi les habitants de son village quitter celui-ci pour se ruer vers la Côte Nord et ses mines, vers Gagnon en particulier, qui se nommait le Lac Jeannine à l'origine. Aujourd'hui, la ville de Gagnon a été rasée, enterrée, fermée, et Lac-au-Saumon survit comme il peut, ayant malgré tout conservé encore son bureau des postes. Mais pour combien de temps, la jeunesse désertant le village en quête d'emplois? En 1975, celui qui s'adresse à vous enseignait à l'UQAM où il était professeur permanent; il a alors choisi, faulknérien du timbre-poste et de stricte observance, de revenir en sa région natale où il laisse ce soir, en accord avec "les eaux de mars" qui coiffent 
l'événement auquel il participe et en accord aussi avec l'épigraphe de son texte choisie en fonction du titre dudit événement - où il laisse ce soir, donc, «déferler le flot qui monte du pays natal ».

Ce "flot de mars" voulant essentiellement dire ceci, qui est en entier accord avec le timbre-poste de Faulkner: le mot région et son adjectif, régionaliste, n'ont rien de péjoratif un pays n'est jamais uniforme, heureusement, toutes ses régions, la nôtre comme celle de Montréal ou de Québec, celle du Mississippi de Faulkner comme celle de New York, ayant leur identité et leurs prérogatives. Les hivers sont longs dans l'Est du Québec, nous dit Montréal? Et alors? New York et Miami disent la même chose de Montréal. Et alors? Les longs hivers, encore plus longs quand ils sont vus depuis Montréal ou Miami, n'ont certes pas empêché la Suède de nous donner Strindberg, Bergman, Volvo et Ikea. Alors? Rappeler qu'un timbre-poste se défend et citer les vers suivants de Sylvain Rivière, écrivain né à Carleton et résidant aux Îles-de-laMadeleine, vers extraits d'un poème intitulé "J'ai pu envie..." qui lui-même fait partie du recueil intitulé Entre le verbe et le patois:

J'ai pu envie de grands sourires

Pour cacher mes larmes au soleil

D'être le Bozo qui fait rire

Et qui pleure dans son sommeil

J'ai pu envie d'être un autre

Qui ne me ressemblerait pas

Fini de jouer le bon apôtre

Ni Dieu ni diable me voilà. 3

Les Ides de mars, prophétiques et malheureuses dans le Jules César de Shakespeare, ne doivent jamais tarir les eaux de mars. Heureusement, la neige a été abondante cet hiver; les eaux de mars (et d'avril) n'en rouleront et n'en tonneront que mieux.

\section{Renald Bérubé, Université du Québec à Rimouski}

3 Sylvain Rivière, Entre le verbe et le patois, Iles-de-la-Madeleine, Marées basses, 1986 , p. 77. 